\title{
The Degree Measure as Utility Function over Positions in Networks
}

Rene (J.R.) van den Brink ${ }^{1}$ Agnieszka Rusinowska ${ }^{2}$ 
Tinbergen Institute is the graduate school and research institute in economics of Erasmus University Rotterdam, the University of Amsterdam and VU University Amsterdam.

Contact: discussionpapers@tinbergen.nl

More TI discussion papers can be downloaded at the Tinbergen Site

Tinbergen Institute has two locations:

Tinbergen Institute Amsterdam

Gustav Mahlerplein 117

1082 MS Amsterdam

The Netherlands

Tel.: +31(0)20 5984580

Tinbergen Institute Rotterdam

Burg. Oudlaan 50

3062 PA Rotterdam

The Netherlands

Tel.: +31(0)10408 8900 


\title{
The degree measure as utility function over positions in networks
}

\author{
René van den Brink ${ }^{\star 1}$ and Agnieszka Rusinowska² \\ 1 Department of Econometrics and Tinbergen Institute, VU University \\ De Boelelaan 1105, 1081 HV Amsterdam, The Netherlands \\ jrbrink@feweb.vu.nl \\ 2 Paris School of Economics - CNRS, University Paris 1, Centre d'Economie de la Sorbonne \\ 106-112 Bd de l'Hôpital, 75647 Paris Cedex 13, France \\ agnieszka.rusinowska@univ-paris1.fr
}

\begin{abstract}
In this paper, we connect the social network theory on centrality measures to the economic theory of preferences and utility. Using the fact that networks form a special class of cooperative TU-games, we provide a foundation for the degree measure as a von Neumann-Morgenstern expected utility function reflecting preferences over being in different positions in different networks. The famous degree measure assigns to every position in a weighted network the sum of the weights of all links with its neighbours. A crucial property of a preference relation over network positions is neutrality to ordinary risk. If a preference relation over network positions satisfies this property and some regularity properties, then it must be represented by a utility function that is a multiple of the degree centrality measure. We show this in three steps. First, we characterize the degree measure as a centrality measure for weighted networks using four natural axioms. Second, we relate these network centrality axioms to properties of preference relations over positions in networks. Third, we show that the expected utility function is equal to a multiple of the degree measure if and only if it represents a regular preference relation that is neutral to ordinary risk. Similarly, we characterize a class of affine combinations of the outdegree and indegree measure in weighted directed networks and deliver its interpretation as a von Neumann-Morgenstern expected utility function.
\end{abstract}

JEL Classification: D81, D85, C02

Keywords: Weighted network, network centrality, utility function, degree centrality, von Neumann-Morgenstern expected utility function, cooperative TU-game, weighted directed network.

\section{Introduction}

The study of network centrality originates from the social network literature where different types of network centrality are distinguished such as degree, closeness, betweenness, prestige, etc. Various centrality measures are developed measuring these types of centrality. More recently, these centrality measures are applied to economic networks. However, there is no utility foundation of network centrality. Since economic decision making is based on preferences of economic decision makers, a utility foundation is fundamental for the application of network centrality measures in economic models. Our aim is to provide such a utility foundation for network centrality by considering network centrality measures as von Neumann-Morgenstern expected utility functions (von Neumann and Morgenstern (1944)) reflecting preferences over positions in networks. In this way, we can

\footnotetext{
* Corresponding author
} 
evaluate different positions in different networks. Questions that can be addressed in this context are, for example, does an agent prefer to be the top of a small organization or a middle manager in a large organization? Notice that centrality only takes the network into account and not the possible (economic) process that takes place on the network. In that sense, centrality as utility only refers to an agent's preference to be in a certain position in the network. If one wishes to analyze networks with different strengths of interaction or relation between two individuals, then weighted networks where each link has a nonnegative weight, can appear more suitable. In this paper we consider such weighted networks.

The present work builds on Roth (1977a) who motivates the Shapley value (Shapley (1953)) as a von Neumann-Morgenstern expected utility function over being particular players in different games. He distinguishes between two kinds of risk: ordinary risk and strategic risk. While ordinary risk involves the uncertainty that arises from lotteries, strategic risk involves the uncertainty that arises from the interaction of the players in a game. Roth (1977a) shows that the Shapley value of a game is equal to the utility of playing the game if and only if the underlying preferences are neutral to both ordinary and strategic risk. From Deng and Papadimitriou (1994), it follows that networks can be seen as a special class of cooperative TU-games, specifically networks are those games where worth is generated only by two-player coalitions.

In the present paper, we apply the method of Roth to give a foundation of social network centrality measures as expected utility functions. A preference relation that evaluates different positions in different networks is a binary relation which for any two network positions (in possibly different networks) states if one of the two positions is (weakly) preferred over the other. Such a preference relation is represented by a utility function if this function assigns values to network positions such that one position is weakly preferred to another if and only if the utility value of the first position is greater or equal to that of the second position. In that sense, mathematically a utility function over network positions is equivalent to a network centrality measure. We state several axioms for preference relations over network positions, and show that a utility function represents regular preferences neutral to ordinary risk if and only if the utilities assigned to network positions are a multiple of their degree, i.e. their number of neighbours. In this way, the degree measure can be seen as a von Neumann-Morgenstern expected utility function for positions in networks. Neutrality to ordinary risk - the crucial axiom that characterizes the degree measure as an expected utility function - means that an agent is indifferent between taking a position in a convex combination of two networks and playing a lottery over the two networks with the corresponding probabilities.

The degree measure is one of the most natural and famous centrality concepts which can be seen as an index of the node's communication ability and assigns to every node in a network its degree. The degree of a node in a weighted network equals the sum of the weights of all links formed by the given node, see e.g. Newman (2010). For simple networks this then boils down to the number of links formed by that node, i.e. the number of its neighbours. Inspired by some experimental studies, Shaw (1954) presents the degree centrality as a measure to be used for predicting the behavior of individuals in small groups, and Nieminen (1974) and van den Brink and Gilles (2000) analyze it from an axiomatic point of view. Although the degree can be seen as a myopic centrality measure that only takes account of direct relations, it can also be considered as a farsighted 
centrality measure in the sense that for a simple connected network, the probability that a random walk will be found at node $i$ in the limit of long time is proportional to the degree of that node, see e.g. Newman (2010) page 159. Besides these myopic and farsighted interpretations, we provide a third interpretation to the degree of nodes in a network as being a von Neumann Morgenstern expected utility function.

The characterization of the degree measure as a von Neumann-Morgenstern expected utility function follows from an axiomatization of the degree measure as a centrality measure for weighted networks using the following four axioms. Anonymity imposes that the labeling of the nodes has no effect on the measure. The isolated node property states that the value assigned to an isolated node (i.e., a node that has no neighbours) does not depend on the structure of the rest of the network. Scale invariance says that if a weighted network is rescaled, i.e., the weights of all its links are multiplied by a common factor, then the measure is also multiplied by the same factor. Additivity states that if we add two weighted networks, then the measure of the obtained 'sum' network is equal to the sum of the measures of the two weighted networks.

Besides undirected networks, we extend the results to directed networks and characterize a class of affine combinations of the outdegree (being the sum of the weights of a node's outgoing arcs) and indegree measures (being the sum of the weights of a node's ingoing arcs) as expected utility function. Directed graphs cannot be represented as TUgames, but they form a special class of generalized TU-games as introduced in Nowak and Radzik (1994).

Related literature As already mentioned, we use the approach of Roth (1977a), building on the utility theory over mixture sets of Herstein and Milnor (1953). Roth (1977a) develops a preference relation over game positions which permits to compare being different players in different games. He extends the preference relation to lotteries over games and shows that the Shapley value is an expected utility function reflecting preferences neutral to both ordinary and strategic risk; see also Roth (1977b,c, 1988) for related studies on this issue. Roth $(1977 \mathrm{c}, 1988)$ considers how the Shapley-Shubik index (Shapley and Shubik (1954)) can be uniquely characterized as a risk-neutral expected utility function defined on the class of simple games. Other attitudes towards risk lead to different utility functions. This concerns, for instance, the Banzhaf index (Banzhaf (1965)) proposed in the context of simple games, and extended to TU-games by e.g. Coleman (1971), Dubey (1975), Dubey and Shapley (1979), and Owen (1975). The Banzhaf index is an extended utility function reflecting preferences averse to strategic risk and neutral to ordinary risk (Roth (1977d)). For an overview of Roth's approach to the Shapley value, see also Pintér (2014).

This paper is also related to the literature on social networks and centrality; for some pioneering articles see e.g. Bavelas (1948), Bavelas (1950), Katz (1953), Beauchamp (1965), Sabidussi (1966), Freeman (1977), Freeman (1979), Bonacich (1972), Bonacich (1987), for surveys see e.g. Borgatti (2005), Goyal (2007), Jackson (2008), and Newman (2010). Our research is particularly related to works using the axiomatic approach to centrality measures. This stream of literature focusses mainly on specific centrality measures. For instance, Garg (2009) characterizes axiomatically the degree, decay and closeness centralities. Some prestige and eigenvector-related centrality measures are characterized in Palacios-Huerta and Volij (2004), Slutzki and Volij (2006), Dequiedt and Zenou (2014), 
and Kitti (2016). Bloch et al. (2016) characterize the standard centrality measures within a unified framework and show that they all are characterized by a common set of axioms. Although the present paper also uses the axiomatic approach to centrality measures and characterizes the degree measure, our main aim is to show that this measure can be interpreted as an expected utility function reflecting preferences over positions in weighted networks.

An issue closely related to centrality is the ranking of nodes which is treated by using a ranking method. Formally, a ranking method assigns to every (weighted) network a (complete) preorder on the set of nodes. This preorder is a ranking of the nodes in order of 'importance' or 'centrality' in the network. Various ranking methods are characterized in the literature, in particular, methods based on directed networks, see e.g. Rubinstein (1980) for the ranking by outdegree on the class of tournaments, see also Henriet (1985) and Bouyssou (1992) for the ranking by Copeland score (Copeland (1951)), Bouyssou and Perny (1992), van den Brink and Gilles (2003) for the ranking by outdegree for arbitrary directed networks, and van den Brink and Gilles (2009) for the outflow ranking method for weighted directed networks. There exist recent studies that characterize ranking methods based on evaluations or citations which consider one-sided settings (e.g. Demange (2014)) and ranking methods in two-sided settings (e.g. Demange (2016)). An important difference between such ranking methods and the topic of this paper is that ranking methods only compare the positions in one and the same network. This is useful if one wants to rank, for example, teams in a sports competition, alternatives in a preference relation, web pages on the internet, etc. Besides such comparisons within one network, a main goal of the underlying paper is to compare positions in different networks. For example, we want to know if an agent prefers a 'central' position in a small network to a position in the fringe of a large network. In order to answer these questions we need to be able to compare positions in different networks.

This paper is organized as follows. In Section 2 we discuss preliminaries on networks and Herstein and Milnor (1953)'s expected utility theory over mixture sets. In Section 3 we present our main result, characterizing the degree measure as a von NeumannMorgenstern expected utility function, using as intermediary results an axiomatization of the degree measure as a centrality measure, and relating properties of network centrality measures to properties of preference relations over network positions. In Section 4 we consider directed networks. Section 5 contains concluding remarks. The paper ends with two appendices: Appendix A on networks as cooperative games and Appendix B containing proofs.

\section{Preliminaries}

In this section we present basic concepts and notation that will be used in the paper.

Weighted networks A weighted undirected network is a pair $(N, \omega)$ consisting of a finite set of nodes $N \subset \mathbb{N}$ that can represent individuals or agents, and a weight function $\omega: L^{c} \rightarrow \mathbb{R}_{+}$, where $L^{c}=\{\{i, j\} \mid i, j \in N, i \neq j\}$ denotes the complete undirected network on $N$. An element $\{i, j\} \in L^{c}$ is a subset of $N$ of size two and is called a link. A link $\{i, j\}$ represents a certain bilateral relationship between nodes $i$ and $j$. A weight 
function gives a nonnegative weight $\omega(\{i, j\})$ to every link that can be interpreted as the 'importance' or 'strength' of that relationship. By $\mathcal{W} \mathcal{G}^{N}$ we denote the collection of all weight functions on $N$. When there is no confusion, in this paper we refer to a weighted undirected network simply as a network. A network with $\omega(\{i, j\}) \in\{0,1\}$ for all $\{i, j\} \in L^{c}$, is usually called a simple network. A simple network just describes the relationships that are present, but says nothing about their intensity. Since $N$ is assumed to be fixed, we represent a network $(N, \omega)$ by the weight function $\omega$.

A centrality measure is a function $f: \mathcal{W} \mathcal{G}^{N} \rightarrow \mathbb{R}^{N}$ that assigns a real number to every node in every network that reflects the 'centrality' of the nodes in the network. The degree of node $i \in N$ in network $\omega$ is defined as the sum of the weights of all links containing $i$, and thus is given by

$$
d_{i}(\omega)=\sum_{j \in N \backslash\{i\}} \omega(\{i, j\})
$$

The degree measure is the centrality measure that assigns to any node $i$ in any network $\omega$ its degree $d_{i}(\omega)$.

Let $\Pi(N)$ be the collection of all permutations $\pi: N \rightarrow N$. For a network $\omega \in$ $\mathcal{W} \mathcal{G}^{N}$ and a permutation $\pi \in \Pi(N)$, the permuted network $\pi \omega \in \mathcal{W} \mathcal{G}^{N}$ is given by $\pi \omega(\{\pi(i), \pi(j)\})=\omega(\{i, j\})$ for every $\{i, j\} \in L^{c}$.

We denote the set of networks where $i \in N$ is an isolated node by

$$
\mathcal{W G}_{i}^{N}=\left\{\omega \in \mathcal{W G}^{N} \mid \omega(\{i, j\})=0 \text { for all } j \in N \backslash\{i\}\right\},
$$

and $\omega^{0} \in \mathcal{W} \mathcal{G}^{N}$ denotes the empty network given by $\omega^{0}(\{i, j\})=0$ for all $i, j \in N$.

By $\omega^{i} \in \mathcal{W} \mathcal{G}^{N}$, we denote the simple star network with $i$ as center given by

$$
\omega^{i}(\{i, j\})=1 \text { for all } j \in N \backslash\{i\} \text { and } \omega^{i}(\{h, j\})=0 \text { if } i \notin\{h, j\} .
$$

Directed networks A weighted directed network is a pair $(N, \omega)$ with $N \subset \mathbb{N}$ and a directed weight function $\omega: N \times N \rightarrow \mathbb{R}_{+}$assigning to every arc $(i, j)$ (i.e., to every ordered pair of nodes) a nonnegative weight $\omega(i, j)$. We only consider weighted directed networks that satisfy $\omega(i, i)=0$ for every $i \in N$. We denote the collection of all directed weight functions on $N$ by $\mathcal{W} \mathcal{D}^{N}$. Similarly as for undirected networks, we refer to a weighted directed network simply as a directed network and represent a weighted directed network $(N, \omega)$ by $\omega$ if there is no confusion.

A centrality measure or power measure for directed networks is a function $f: \mathcal{W D}^{N} \rightarrow$ $\mathbb{R}^{N}$ that assigns a real number to every node in every directed network. The outdegree of node $i$ in weighted directed network $\omega$ is the sum of the weights of $i$ 's outgoing arcs, and thus is defined by

$$
\text { out }_{i}(\omega)=\sum_{j \in N} \omega(i, j)
$$

The indegree of node $i$ in weighted directed network $\omega$ is the sum of the weights of its ingoing arcs, and is given by

$$
i n_{i}(\omega)=\sum_{j \in N} \omega(j, i)
$$


The outdegree-, respectively indegree measure is the centrality measure that assigns to any node $i$ in any directed network $\omega$ its outdegree out $_{i}(\omega)$, respectively its indegree $i n_{i}(\omega)$.

Note that both measures generalize the degree measure in the sense that, representing a undirected network by the directed network where every link $\{i, j\}$ is represented by two $\operatorname{arcs}(i, j),(j, i)$ oriented in opposite direction, yields that both the outdegree as well as the indegree of a node in the associated directed network, equals its degree in the undirected network. ${ }^{1}$ For a directed network $\omega \in \mathcal{W D}^{N}$ and a permutation $\pi \in \Pi(N)$, the permuted directed network $\pi \omega \in \mathcal{W D}^{N}$ is given by $\pi \omega(\pi(i), \pi(j))=\omega(i, j)$ for every $(i, j) \in N \times N$.

We denote the set of weighted directed networks where $i$ is an isolated node by

$$
\mathcal{W D}_{i}^{N}=\left\{\omega \in \mathcal{W} \mathcal{D}^{N} \mid \omega(i, j)=\omega(j, i)=0 \text { for all } j \in N \backslash\{i\}\right\},
$$

and $\omega^{0} \in \mathcal{W} \mathcal{D}^{N}$ denotes the empty directed network given by $\omega^{0}(i, j)=0$ for all $i, j \in N$. Furthermore, $\omega^{i} \in \mathcal{W} \mathcal{D}^{N}$ is the standard outward oriented star network with $i$ as center given by $\omega^{i}(i, j)=1$ for all $j \in N \backslash\{i\}$, and $\omega^{i}(h, j)=0$ otherwise.

Note that we use the same notations $\omega, \omega^{0}$ and $\omega^{i}$ for both undirected and directed networks. However, $\omega(\{i, j\})$ and $\omega(i, j)$ clarifies whether we consider networks or directed networks. Moreover, the cases of undirected and directed networks are presented in separate sections, i.e., in Sections 3 and 4, respectively, and therefore it will be clear from the context if we deal with $\mathcal{W} \mathcal{G}^{N}$ or $\mathcal{W} \mathcal{D}^{N}$.

Expected utility We recapitulate the utility theory on mixture sets of Herstein and Milnor (1953) (for some related works and literature on linear utility representation theorems, see e.g., Trockel (1989), Trockel (1992), and Neuefeind and Trockel (1995)). Consider a set $M$. The lottery between two elements $a, b \in M$ where element $a$ occurs with probabilty $p \in[0,1]$ is denoted by $[p a ;(1-p) b]$. A set $M$ is a mixture set if for any $a, b \in M$ and any $p \in[0,1]$, the lottery $[p a ;(1-p) b]$ also belongs to $M$. Notice that this implies that also all loteries over loteries etc. belong to $M$. It is assumed that for all $a, b \in M$ and $p, q \in[0,1]$, the following standard equalities hold:

$$
[1 a ; 0 b]=a, \quad[p a ;(1-p) b]=[(1-p) b ; p a], \quad[q[p a ;(1-p) b] ;(1-q) b]=[p q a ;(1-p q) b] .
$$

A preference relation on $M$ is a binary relation $\succeq$ with the interpretation that $a \succeq b$ means that " $a$ is at least as good as $b$ ".

A function $u: M \rightarrow \mathbb{R}$ is an expected utility function representing the preference relation $\succeq$ if for all $a, b \in M$ and $p \in[0,1]$, it holds that

$$
\begin{aligned}
& \text { (i) } u(a) \geq u(b) \text { if and only if } a \succeq b \text {, and } \\
& \text { (ii) } u([p a ;(1-p) b])=p u(a)+(1-p) u(b) .
\end{aligned}
$$

We write $[a \succ b]$ if and only if $[a \succeq b$ and $b \nsucceq a]$, and $[a \sim b]$ if and only if $[a \succeq b$ and $b \succeq a]$.

The following axioms guarantee that an expected utility function representing $\succeq$ exists.

\footnotetext{
${ }^{1}$ Another famous measure for directed networks is the Copeland score (Copeland (1951)) which assigns to every directed network the outdegree minus the indegree. Although often applied, this is not a generalization of the degree measure for undirected networks.
} 
Axiom 1 (Completeness) For any $a, b \in M$ either $a \succeq b$ or $b \succeq a$.

Axiom 2 (Transitivity) For any $a, b, c \in M$ such that $a \succeq b$ and $b \succeq c$ it holds that $a \succeq c$.

Axiom 3 (Continuity) For any $a, b, c \in M$, the sets $\{p \mid[p a ;(1-p) b] \succeq c\}$ and $\{p \mid$ $c \succeq[p a ;(1-p) b]\}$ are closed.

Axiom 4 (Substitutability) If $a, a^{\prime} \in M$ and $a \sim a^{\prime}$ then for any $b \in M,\left[\frac{1}{2} a ; \frac{1}{2} b\right] \sim$ $\left[\frac{1}{2} a^{\prime} ; \frac{1}{2} b\right]$.

The expected utility function that represents $\succeq$ is unique up to an affine transformation. Choose $r_{0}, r_{1} \in M$ such that $r_{1} \succ r_{0}$. For any $x \in M$, we can write

$$
u(x)=\frac{p_{a b}(x)-p_{a b}\left(r_{0}\right)}{p_{a b}\left(r_{1}\right)-p_{a b}\left(r_{0}\right)}
$$

where $a, b \in M$ are such that $a \succeq x \succeq b$ and $a \succeq r_{1} \succ r_{0} \succeq b$, and $p_{a b}(x)$ is defined by

$$
x \sim\left[p_{a b}(x) a ;\left(1-p_{a b}(x)\right) b\right],
$$

i.e. $p_{a b}(x)$ is the probability on $a$ in the lottery between $a$ and $b$ such that the agent is indifferent between this lottery and getting $x$ for sure. Note that the $p_{a b}$ are well defined and $u$ is independent of the choice of $a$ and $b$. Moreover, $r_{1}$ and $r_{0}$ determine the origin and scale of the utility function: $u\left(r_{1}\right)=1$ and $u\left(r_{0}\right)=0$. We assume throughout the paper that preferences satisfy the axioms stated above.

\section{Centrality and utility in undirected networks}

We refer to a pair $(i, \omega) \in N \times \mathcal{W G}^{N}$ as a network position. We assume that a preference relation $\succeq$ is defined on the set $N \times \mathcal{W} \mathcal{G}^{N}$ of network positions. We interpret $(i, \omega) \succeq\left(j, \omega^{\prime}\right)$ as "it is at least as good to be in the position of node $i$ in network $\omega$ as to be in the position of node $j$ in network $\omega^{\prime \prime \prime}$. Let $M$ be the mixture set generated by all network positions $(i, \omega) \in N \times \mathcal{W G}^{N}$, so $M$ contains all network positions $(i, \omega)$, all lotteries of network positions, all lotteries over those lotteries, etc. For $(i, \omega),\left(j, \omega^{\prime}\right) \in N \times \mathcal{W} \mathcal{G}^{N}$ and $p \in[0,1]$, the lottery $\left[p(i, \omega) ;(1-p)\left(j, \omega^{\prime}\right)\right]$ considers a type of risk with respect to taking a position in a network. It means that with probability $p$ the agent takes the position of node $i$ in network $\omega$, and with probability $(1-p)$ he takes the position of node $j$ in network $\omega^{\prime}$. We extend the preference relation over network positions to the mixture set $M$. Then an expected utility function for network positions is a function $\phi: M \rightarrow \mathbb{R}$ assigning a utility value to every mixture of network positions.

Using the fact that networks can be seen as a special class of cooperative TU-games (see Deng and Papadimitriou (1994)), besides the standard axioms on mixture sets stated in the preliminaries (Axioms 1 - 4), we apply some of the axioms of Roth (1977a) on this special class of so-called 2-games. ${ }^{2}$ (We refer to Appendix A for this game representation of networks. We will state all axioms and results in terms of network positions.)

The first requires that relabeling the nodes in a network yields a corresponding reordering in the preference relation.

\footnotetext{
${ }^{2}$ Axioms 5 and 7 are exactly the same as formulated by Roth (1977a) but restricted to the class of 2-games. Axiom 6 is a modification of Roth's corresponding axiom.
} 
Axiom 5 For all $\omega \in \mathcal{W G}^{N}, i \in N$ and $\pi \in \Pi(N)$, it holds that $(i, \omega) \sim(\pi(i), \pi \omega)$.

The second axiom compares different network positions, expressing preference with respect to connectedness. More specifically, an agent (i) weakly prefers any position in any network above being in the empty network, (ii) is indifferent between being isolated in any network and being in the empty network, and (iii) strictly prefers to be the center of the star than being in the empty network. ${ }^{3}$

Axiom 6 For all $i \in N, \omega \in \mathcal{W} \mathcal{G}^{N}$ and $\omega^{\prime} \in \mathcal{W} \mathcal{G}_{i}^{N}$, it holds that (i) $(i, \omega) \succeq\left(i, \omega^{0}\right)$, (ii) $\left(i, \omega^{\prime}\right) \sim\left(i, \omega^{0}\right)$, and $($ iii $)\left(i, \omega^{i}\right) \succ\left(i, \omega^{0}\right)$.

This axiom expresses the importance of being connected in the sense that the worst that can happen is to be isolated. Note that condition (iii) does not imply that it is best to be the center of a star but, together with part (ii) it implies that it is strictly better to be the center of a star than to be isolated. This rules out externalities in the sense that as long as an agent is isolated, it is indifferent with respect to the way the other nodes are connected. Although from an economic viewpoint there might be reason why such externalities exist, the goal of this paper is to view centrality measures as utility functions, and centrality measures in the social network literature usually assign centrality zero to isolated positions. Concerning part (iii), whatever type of centrality is considered (degree, connectedness, betweenness etc.), on a fixed set of nodes, it is always more central to be the center of a star than to be isolated. ${ }^{4}$

From now on, we refer to preference relations that satisfy Axioms 1 - 6 as regular preference relations.

To axiomatize the Shapley value for TU-games as a von Neumann-Morgenstern expected utility function, Roth (1977a) introduces two types of risk neutrality: neutrality to ordinary risk and neutrality to strategic risk. In this paper, we need to consider only the first type of risk. For $p \in[0,1]$, consider node $i$ in a convex combination $p \omega+(1-p) \omega^{\prime}$ of two networks $\omega$ and $\omega^{\prime}$, i.e. $\left(\omega+\omega^{\prime}\right)(\{i, j\})=\omega(\{i, j\})+\omega^{\prime}(\{i, j\})$ for all $i, j \in N$. Neutrality to ordinary risk requires that an agent is indifferent between taking a position in network $p \omega+(1-p) \omega^{\prime}$ for sure, and playing a lottery over the networks $\omega$ and $\omega^{\prime}$ with the corresponding probabilities.

Axiom 7 (Neutrality to ordinary risk) For all $\omega, \omega^{\prime} \in \mathcal{W} \mathcal{G}^{N}$ and $i \in N$, it holds that $\left(i, p \omega+(1-p) \omega^{\prime}\right) \sim\left[p(i, \omega) ;(1-p)\left(i, \omega^{\prime}\right)\right]$.

Next, we state the main result of this section characterizing the class of expected utility functions that represent a regular preference relation that is neutral to ordinary risk as those that correspond to a multiple of the degree measure.

\footnotetext{
3 This axiom is similar to Condition 7 of Roth (1977a) in the sense that (i) the 2-game associated to a network is the null game if and only if the network is the empty network, (ii) a player is a null player in the associated 2-game if and only if it is isolated in the network, and (iii) whereas Roth (1977a) required that is is strictly preferred to be a dictator in a simple game (i.e. all coalitions containing player $i$ earn one, and all other coalitions earn zero) than to be in the null game, here the center of a star is not a dictator but a veto player in the 2-game where the payoff is generated by all two-player coalitions containing this player.

${ }^{4}$ Our results are also valid if this axiom is strengthened by requiring that being the center of the simple star is strictly better than any other position in any simple network on a fixed set of positions. This is often required when measuring centrality in the social network literature, see e.g. Gómez et al. (2003).
} 
Theorem 1 The expected utility function $\phi$ represents a regular preference relation that is neutral to ordinary risk if and only if there exists an $\alpha>0$ such that $\phi(i, \omega)=\alpha d_{i}(\omega)$ for all $(i, \omega) \in N \times \mathcal{W G}^{N}$, where $d_{i}(\omega)$ is the degree of node $i$ in network $\omega$, see (1).

This theorem gives the degree measure, which is a well-known centrality measure in social network theory, an interpretation as a von Neumann-Morgenstern expected utility function. This can be shown by (i) characterizing (the class of multiples of) the degree measure as those centrality measures that satisfy four properties of centrality measures for networks (Proposition 1), and (ii) relating those four network centrality properties to properties of preference relations over network positions (Lemma 1).

Note that centrality measures $f$ and utility functions $\phi$ are mathematically the same and both assign values to nodes in networks, but their interpretation is different. It is the purpose of this paper to bring these interpretations together.

Realizing that networks are equivalent to cooperative TU-games where the worth of any coalition is equal to the sum of the worths of all its subsets of size two, one can apply any axiomatization of the Shapley value on this class of 2-games, obtaining an axiomatization of the degree measure as a centrality measure if uniqueness holds on the subclass. Here we verify axioms similar as Shapley (1953)'s axioms (without efficiency).

First, anonymity means that the labeling of the nodes in a network has no effect on their centrality.

Property 1 (Anonymity) For every $\omega \in \mathcal{W} \mathcal{G}^{N}$ and permutation $\pi \in \Pi(N)$, it holds that $f_{i}(\omega)=f_{\pi(i)}(\pi(\omega))$.

Second, the isolated node property requires that the centrality of an isolated node does not depend on the structure of the rest of the network. Centrality measures from the literature usually satisfy this property.

Property 2 (Isolated node property) For every $\omega, \omega^{\prime} \in \mathcal{W} \mathcal{G}_{i}^{N}$, it holds that $f_{i}(\omega)=$ $f_{i}\left(\omega^{\prime}\right)$.

Scale invariance states that if the weights of all links in a network are multiplied by a common factor, then the centralities of the nodes in that network are multiplied by the same factor.

Property 3 (Scale invariance) Let $\omega, \omega^{\prime} \in \mathcal{W} \mathcal{G}^{N}$ be such that there exists an $\alpha \in \mathbb{R}_{+}$ with $\omega^{\prime}(\{i, j\})=\alpha \cdot \omega(\{i, j\})$ for all $\{i, j\} \in L^{c}$. Then $f\left(\omega^{\prime}\right)=\alpha f(\omega)$.

Finally, additivity means that the centralities in the network obtained by adding two networks is equal to the sum of the centralities of these two networks.

Property 4 (Additivity) For $\omega, \omega^{\prime} \in \mathcal{W} \mathcal{G}^{N}$ it holds that $f\left(\omega+\omega^{\prime}\right)=f(\omega)+f\left(\omega^{\prime}\right)$, where $\left(\omega+\omega^{\prime}\right)(\{i, j\})=\omega(\{i, j\})+\omega^{\prime}(\{i, j\})$ for all $\{i, j\} \in L^{c}$.

Since the Shapley value of the 2-game associated to a network assigns to every player half of its degree (see Deng and Papadimitriou (1994), Theorem 1), we obtain the following characterization of the (multiples of the) degree measure. 
Proposition 1 A centrality measure $f$ satisfies anonymity, the isolated node property, scale invariance and additivity if and only if there exists an $\alpha \in \mathbb{R}$ such that

$$
f_{i}(\omega)=\alpha d_{i}(\omega) \text { for all }(i, \omega) \in N \times \mathcal{W} \mathcal{G}^{N} .
$$

Theorem 1 follows from Proposition 1 by the following lemma which shows how the four properties for network centrality measures are implied by the regularity Axioms 5 6 and neutrality to ordinary risk (Axiom 7) on preferences.

Lemma 1 Consider an expected utility function $\phi: M \rightarrow \mathbb{R}$ for positions in a network that is determined by a centrality measure $f$ as follows: $\phi(i, \omega)=f_{i}(\omega)$.

(i) If expected utility function $\phi$ represents a preference relation $\succeq$ satisfying Axiom 5 , then centrality measure $f$ satisfies anonymity.

(ii) If expected utility function $\phi$ represents a preference relation $\succeq$ satisfying Axiom 6 , then centrality measure $f$ satisfies the isolated node property.

(iii) If expected utility function $\phi$ represents a preference relation that is neutral to ordinary risk (Axiom 7), then for all $\omega \in \mathcal{W G}^{N}, i \in N$ and $c>1$, it holds that $(i, \omega) \sim\left[\frac{1}{c}(i, c \omega) ;\left(1-\frac{1}{c}\right)\left(i, \omega^{0}\right)\right]$.

(iv) If expected utility function $\phi$ represents a preference relation $\succeq$ satisfying Axioms 5-6 and is neutral to ordinary risk (Axiom 7), then centrality measure $f$ satisfies scale invariance and additivity.

This lemma and the main theorem also can be shown similar as Roth (1977a), realizing that networks form a special class of cooperative TU-games. We will prove a similar result for directed networks in Appendix B.

Our main result (Theorem 1) is an economic result interpreting such centrality measures as von Neumann-Morgenstern expected utility functions. Proposition 1 characterizes the (multiples of the) degree measure as a social network centrality measure. To prove Theorem 1 from Proposition 1, we needed Lemma 1 which is a result that 'bridges' social network theory with economic utility theory.

\section{Centrality and utility in directed networks}

In this section, we extend the utility foundation of centrality measures as done in the previous section to directed networks, where they are also sometimes called power measures. Directed networks cannot be represented as a special class of cooperative TU-games. In order to describe directed networks as cooperative games, we need a game model where the worth of coalitions depends on the order of the players in the coalition. For example, the order/arc $(1,2)$ can have a different worth/weight than the order $(2,1)$, whereas in a TU-game/undirected network, the order in coalition $\{1,2\}=\{2,1\}$ does not matter. We do this by considering a subclass of generalized characteristic functions as introduced in Nowak and Radzik (1994) where only orders of length 2 have a nonzero worth. We refer to Appendix A for a summary of this model, but here we apply the notions directly on directed networks. However, since the theory of Roth (1977a) is not yet extended to the class of generalized characteristic functions, we give all proofs for directed networks in Appendix B. 
In this section we consider the set $N \times \mathcal{W} \mathcal{D}^{N}$ of positions in weighted directed networks. Similar as in the previous section, we now assume that a preference relation $\succeq$ is defined on the set $N \times \mathcal{W D}^{N}$ with the interpretation $(i, \omega) \succeq\left(j, \omega^{\prime}\right)$ as "it is at least as good to be in position $i$ in directed network $\omega$ than to be in position $j$ in directed network $\omega^{\prime \prime \prime}$. We extend this preference relation to the mixture set $M$ that also contains all lotteries $\left[p(i, \omega) ;(1-p)\left(j, \omega^{\prime}\right)\right]$ with $(i, \omega),\left(j, \omega^{\prime}\right) \in N \times \mathcal{W D}^{N}$ and $p \in[0,1]$.

Besides the standard axioms on mixture sets stated in the preliminaries (Axioms 1 - 4) on mixture sets, we use Axioms 5-6-7) of the previous section, but now for directed networks. Notice that the Axioms 8 - 10 are stated exactly the same as these axioms in the previous section, except defined on the class of directed networks $\mathcal{W D}^{N}$, and the star in undirected networks replaced by the outward oriented star in directed networks.

Axiom 8 For all $\omega \in \mathcal{W D}^{N}, i \in N$ and $\pi \in \Pi(N)$, it holds that $(i, \omega) \sim(\pi(i), \pi \omega)$.

Axiom 9 For all $i \in N, \omega \in \mathcal{W} \mathcal{D}^{N}$ and $\omega^{\prime} \in \mathcal{W} \mathcal{D}_{i}^{N}$, it holds that (i) $(i, \omega) \succeq\left(i, \omega^{0}\right)$, (ii) $\left(i, \omega^{\prime}\right) \sim\left(i, \omega^{0}\right)$, and (iii) $\left(i, \omega^{i}\right) \succ\left(i, \omega^{0}\right)$.

Axiom 10 (Neutrality to ordinary risk) For all $\omega, \omega^{\prime} \in \mathcal{W D}^{N}$ and $i \in N$, it holds that $\left(i, p \omega+(1-p) \omega^{\prime}\right) \sim\left[p(i, \omega) ;(1-p)\left(i, \omega^{\prime}\right)\right]$, where $\left(p \omega+(1-p) \omega^{\prime}\right)(i, j)=p \omega(i, j)+$ $(1-p) \omega^{\prime}(i, j)$ for all $(i, j) \in N \times N$.

Similar as in Section 3, we refer to preference relations that satisfy Axioms 8 and 9 as regular preference relations.

It turns out that a utility function for directed network positions that represents regular preferences that are neutral to ordinary risk must be an affine combination of the outdegree and indegree measures with positive weight on the outdegree and nonnegative weight on the indegree measure.

Theorem 2 The utility function $\phi$ represents a regular preference relation that is neutral to ordinary risk if and only if there exist $\alpha>0$ and $\beta \geq 0$ such that $\phi(i, \omega)=\alpha \cdot$ out $_{i}(\omega)+$ $\beta \cdot i n_{i}(\omega)$ for all $(i, \omega) \in N \times \mathcal{W D}^{N}$.

The class of utility functions/centrality measures characterized in this theorem contains the outdegree $(\alpha=1, \beta=0)$ and the average of the out- and indegree $(\alpha=\beta=$ $\frac{1}{2}$ ). From the various extensions of the Shapley value to generalized TU-games, a multiple of the outdegree measure is obtained by applying the extension of Nowak and Radzik (1994), while a multiple of the average of the outdegree and indegree is obtained by applying the alternative generalization of the Shapley value of Sánchez and Bergantinos (1997), see Appendix A.

Similar as in the previous section, Theorem 2 can be shown by characterizing the outdegree measure as a centrality or power measure for directed networks.

Proposition 2 A centrality measure $f$ on $\mathcal{W D}^{N}$ satisfies

- Anonymity: For every $\omega \in \mathcal{W D}^{N}$ and permutation $\pi \in \Pi(N)$, it holds that $f_{i}(\omega)=$ $f_{\pi(i)}(\pi(\omega))$;

- Scale invariance: Let $\omega, \omega^{\prime} \in \mathcal{W D}^{N}$ be such that there exists an $\alpha \in \mathbb{R}$ such that $\omega^{\prime}(i, j)=\alpha \omega(i, j)$. Then $f\left(\omega^{\prime}\right)=\alpha f(\omega)$; 
- Isolated node property: For all $\omega, \omega^{\prime} \in \mathcal{W D}_{i}^{N}$ and $i \in N$, it holds that $f_{i}(\omega)=f_{i}\left(\omega^{\prime}\right)$;

- Additivity: For $\omega, \omega^{\prime} \in \mathcal{W D}^{N}$ it holds that $f\left(\omega+\omega^{\prime}\right)=f(\omega)+f\left(\omega^{\prime}\right)$,

if and only if there exist $\alpha, \beta \in \mathbb{R}$ such that

$$
f_{i}(\omega)=\alpha \cdot \text { out }_{i}(\omega)+\beta \cdot \text { in }_{i}(\omega) \text { for all }(i, \omega) \in N \times \mathcal{W D}^{N} .
$$

Similar as Lemma 1, the axioms for centrality measures can be associated to properties of preference relations.

Lemma 2 Consider a utility function $\phi: N \times \mathcal{W D}^{N} \rightarrow \mathbb{R}$ for positions in a directed network that is determined by a centrality measure $f$ as follows: $\phi(i, \omega)=f_{i}(\omega)$.

(i) If expected utility function $\phi$ represents a preference relation $\succeq$ satisfying Axiom 8 , then centrality measure $f$ satisfies anonymity.

(ii) If expected utility function $\phi$ represents a preference relation $\succeq$ satisfying Axiom 9, then centrality measure $f$ satisfies the isolated node property.

(iii) If expected utility function $\phi$ represents a preference relation that is neutral to ordinary risk (Axiom 10), then for all $\omega \in \mathcal{W D}^{N}, i \in N$ and $c>1$, it holds that $(i, \omega) \sim\left[\frac{1}{c}(i, c \omega) ;\left(1-\frac{1}{c}\right)\left(i, \omega^{0}\right)\right]$.

(iv) If expected utility function $\phi$ represents a preference relation $\succeq$ satisfying Axioms 8-9 and is neutral to ordinary risk (Axiom 10), then centrality measure $f$ satisfies scale invariance and additivity.

The proofs of Proposition 2, Lemma 2 and Theorem 2 can be found in Appendix B.

In this section we characterized a class of affine combinations of the outdegree and indegree measures as von Neumann-Morgenstern expected utility functions for positions in directed networks with a positive weight on the outdegree and a nonnegative weight on the indegree. The signs of the weights are determined by Axiom 9. A position in the 'empty network' being the worst determines that the weights on out- and indegree are nonnegative. The centre of the outward oriented star being better than a position in the 'empty network' determines the weight on the outdegree to be positive. Adding more axioms we can refine the weights. For example, additionally requiring that being the centre of the outward oriented star is strictly better than being the centre of the inward oriented $\operatorname{star}^{5}$ will determine that $\alpha>\beta$, i.e. the weight on the outdegree is greater than the weight on the indegree.

\section{Concluding remarks}

The goal of this paper is to give a utility foundation to centrality measures, and in that way to connect social network theory with economic utility theory. Summarizing,

(i) we showed that an expected utility function $\phi$ represents a regular preference relation that is neutral to ordinary risk if and only if it is a nonnegative multiple of the degree measure for undirected networks (Theorem 1) / a nonnegative affine combination of the outdegree and indegree measure with positive weight on the outdegree for directed networks (Theorem 2);

\footnotetext{
${ }_{5}$ The simple inward oriented star is the directed network given by $\omega_{i}(j, i)=1$ for all $j \in N \backslash\{i\}$, and $\omega_{i}(h, j)=0$ otherwise.
} 
(ii) we showed that a centrality measure $f$ satisfies anonymity, scale invariance, the isolated node property and additivity if and only if it is a multiple of the degree measure for undirected networks (Proposition 1) / an affine combination of the outdegree and indegree measure for directed networks (Proposition 2);

(iii) we related properties of preference relations to properties of centrality measures (Lemma 1 / Lemma 2).

These three steps summarize the connection that we make between economics and social networks. Our main results are Theorems 1 and 2 being economic results which characterize centrality measures as von Neumann-Morgenstern expected utility functions. Propositions 1 and 2 are social network results which give axiomatic characterizations of centrality measures. Finally, Lemmas 1 and 2 bridge economics with social networks by relating properties of economic preference relations to properties of social network centrality measures.

The approach followed in this paper is of crucial importance, since the interpretation of the degree measure as expected utility function for network positions permits to compare different positions in networks. Despite its simplicity, the degree measure is sufficient for measuring involvement or communication ability of an agent in the network. Moreover, the simplicity of the degree measure is an advantage, since only the local structure around a node must be known for calculations, for instance, when using social survey data. Our utility interpretation is also an alternative to the usual interpretation of the degree as either a myopic centrality measure that only takes account of direct relations, or as a farsighted centrality measure in the sense that for a simple connected network, the probability that a random walk will be found at node $i$ in the limit of long time is proportional to the degree of that node.

We plan a number of follow-up research projects. While in this paper the set of nodes is assumed to be fixed, we could consider utility functions over nodes in networks of different size. Another interesting extension would be to analyze processes on a network and to combine utility of positions in a network with utility generation from processes on a network. Different centrality measures usually capture complementary aspects of an agent's position and ability, and therefore can get different agents as the most central ones in the network. We intend to relax the assumption of risk neutrality to find utility foundations of other centrality measures.

We mentioned that the class of all networks can be seen as a subclass of the class of all TU-games, specifically the class of TU-games where only coalitions of size two have a nonzero Harsanyi dividend (Harsanyi (1959)). These games are equivalent to the 2-games or telecommunication games used in van den Nouweland et al. (1996) to model cooperation among national telephone operators to establish the Terrestrial Flight Telephone System, and contains the class of queueing games of Maniquet (2003). An interesting question for future research is if we can obtain utility foundations of other centrality measures by considering other TU-games that represent a network. For example, van den Brink and Gilles (2000) consider the game where every coalition of nodes is assigned the sum of the worths of all links that have at least one player in the coalition. Obviously, the worth of a singleton equals its degree. Applying the Shapley value to this game yields the $\beta$-measure which is another centrality measure. In our future research 
we would like to apply the approach followed in this paper to obtain utility foundations of this and other measures.

Another theoretical question that we want to address is how to incorporate externalities in measuring network centrality. For example, does your utility depend on the way how agents in other components of the network (i.e. agents with whom you are not connected, not directly nor indirectly) are linked? According to centrality measures that can be found in the literature, usually the centrality of a position does not depend on the structure of other components. For example, isolated nodes usually have zero centrality irrespective of the rest of the network. But when you have no neighbors, it might still make a difference whether other agents are linked to each other or not. We plan to incorporate this type of externality in measuring network centrality, in particular when interpreting these measures as utility functions. Besides this theoretical research, we plan to do an experimental study, both testing measures of centrality with and without externalities.

Finally, considering directed networks, there are more possibilities to consider what is the worst possible position, even when there are no externalities. For example, under the regularity axioms of this paper, the utility function assigns zero to every isolated node in any network. For undirected networks this means that being isolated is the worst position (as reflected by Axiom 6). However, for directed networks, it is not obvious whether it is worse to be isolated or to be connected but connected only by having 'superiors' and no 'subordinates', i.e. having only ingoing arcs. This is reflected by the famous Copeland score (Copeland (1951)) being the difference between the outdegree and indegree. Which position is more preferred depends on the application of the network. In some cases it might be better to be connected, even with only ingoing arcs. But in other cases it might be better to be isolated and independent than to be connected with only ingoing arcs.

\section{Acknowledgements}

This research has been initiated when René van den Brink was Visiting Professor at the Centre d'Economie de la Sorbonne of the University of Paris 1. Agnieszka Rusinowska acknowledges the support by the National Agency for Research (Agence Nationale de la Recherche), Project DynaMITE (ANR-13-BSH1-0010-01). Both authors acknowledge the support by the Labex OSE (ANR-10-LABX-93-01). They thank particularly Stefano Moretti for pointing out the relation between networks and cooperative TU-games, and participants of the following conferences, workshops and invited seminars: Workshop on Cooperative Game Theory in Business Practice (Leipzig, June 2016), SING12 Conference (Odense, July 2016), NSF Conference on Network Science in Economics (St Louis, April 2017), CTN Conference (Glasgow, May 2017), SAET Conference (Faro, June 2017), PET Conference (Paris, July 2017), Conference on Game Theory in Stony Brook (July 2017), invited seminars at University of Bayreuth and University of Paris 1.

\section{Appendix A: Cooperative TU-games}

The Shapley value and 2-games A situation in which a finite set of players $N \subset \mathbb{N}$ can generate certain payoffs by cooperation can be described by a cooperative game with transferable utility (or simply a TU-game), being a pair $(N, v)$ where $v: 2^{N} \rightarrow \mathbb{R}$ is a characteristic function on $N$ satisfying $v(\emptyset)=0$. For every coalition $S \subseteq N, v(S) \in \mathbb{R}$ is 
the worth of coalition $S$, i.e. the members of coalition $S$ can obtain a total payoff of $v(S)$ by agreeing to cooperate. Since the set of players/nodes is fixed, we represent a TU-game $(N, v)$ by its characteristic function $v$.

A payoff vector for game $v$ on $N$ is an $|N|$-dimensional vector $x \in \mathbb{R}^{N}$ assigning a payoff $x_{i} \in \mathbb{R}$ to any player $i \in N$. A (single-valued) solution for TU-games is a function $f$ that assigns a payoff vector $f(v) \in \mathbb{R}^{N}$ to every TU-game $v$ on $N$. One of the most famous solutions for TU-games is the Shapley value (Shapley (1953)) given by

$$
S h_{i}(v)=\sum_{S \subseteq N: i \in S} \frac{(|S|-1) !(|N|-|S|) !}{|N| !}(v(S)-v(S \backslash\{i\})) .
$$

Deng and Papadimitriou (1994) argue that any network can be represented by a TU-game $v$ where the worth of any two player coalition is the weight of the link, and the worth of any other coalition equals the sum of the worths of all two player subcoalitions. In other words, the TU-game $v^{\omega}$ associated to network $\omega$ is given by $v^{\omega}(S)=\sum_{T \subseteq S,|T|=2} v(T) .{ }^{6}$ As Deng and Papadimitriou (1994) show, the Shapley value of the associated game $v^{\omega}$ assigns to every player half of its degree in $\omega$.

In van den Nouweland et al. (1996) and Brown and Housman (1988), these games are called 2-games, and it is shown that on this class of games the Shapley value coincides with other TU-game solutions such as the nucleolus (Schmeidler (1969)) and the $\tau$-value (Tijs (1981)).

Generalized TU-games Nowak and Radzik (1994) introduced the concept of game in generalized characteristic function form or generalized TU-game where the order in which a coalition is formed influences the worth that can be generated. For each $S \in 2^{N} \backslash\{\emptyset\}$, let $\Pi(S)$ denote the set of all permutations or ordered coalitions of the players in $S$ and, for notational convenience, $\Pi(\emptyset)=\{\emptyset\}$. We denote $\Omega(N)=\{T \in \Pi(S) \mid S \subseteq N\}$ as the set of all ordered coalitions with players in $N$. A game in generalized characteristic function form is a pair $(N, v)$ with $N$ being the player set and $v: \Omega(N) \rightarrow \mathbb{R}$ being the generalized characteristic function satisfying $v(\emptyset)=0$.

Given an ordered coalition $T \in \Omega(N)$, there exists $S \subseteq N$ such that $T \in \Pi(S)$. We denote by $H(T)=S$ the set of players in the ordered coalition $T$, and $t=|H(T)|$. For ordered coalition $T \in \Omega(N)$, let $j(T) \in\{1, \ldots, t\}$ be the position of player $j \in H(T)$ in $T$. So, for $T=\left(i_{1}, i_{2}, \ldots, i_{t}\right)$ we have $i_{k}(T)=k$ for all $k \in\{1, \ldots, t\}$. For player $i \in N \backslash H(T)$, let $\left(T, i^{l}\right), l \in\{1, \ldots, t+1\}$, be the ordered coalition that is obtained from $T$ by putting $i$ on the $l^{\text {th }}$ position and moving all players on position $l$ or higher one position backwards. So, $i\left(T, i^{l}\right)=l, j\left(T, i^{l}\right)=j(T)$ for all $j \in H(T)$ with $j(T) \in\{1, \ldots, l-1\}$, and $j\left(T, i^{l}\right)=j(T)+1$ for all $j \in H(T)$ with $j(T) \in\{l, \ldots, t\}$. Since the set of players/nodes is fixed, we represent a generalized TU-game $(N, v)$ by its characteristic function $v$.

A solution for generalized TU-games is a function that assigns to every generalized TU-game $v$ a vector in $\mathbb{R}^{N}$. In their seminal paper, Nowak and Radzik (1994) define and characterize the solution $\psi^{N R}$ assigning to every generalized TU-game $v$ the payoffs

$$
\psi_{i}^{N R}(v)=\sum_{T \in \Omega(N \backslash\{i\})} \frac{(n-t-1) !}{n !}\left(v\left(T, i^{t+1}\right)-v(T)\right), \text { for all } i \in N,
$$

\footnotetext{
${ }^{6}$ In terms of the so-called Harsanyi dividends (Harsanyi (1959)) this can also be defined as the class of games where only two-player coalitions have a nonzero dividend being equal to the weight of the corresponding link.
} 
so a player gets its average marginal contribution to the players that entered before him over all orders of $N$. Sánchez and Bergantinos (1997) consider another generalization of the Shapley value for generalized TU-games given by

$$
\psi_{i}^{S B}(v)=\sum_{T \in \Omega(N \backslash\{i\})} \frac{(n-t-1) !}{n !(t+1)} \sum_{l=1}^{t+1}\left(v\left(T, i^{l}\right)-v(T)\right) \text {, for all } i \in N .
$$

which also takes account of the contribution of a player in an ordered coalition where it is not the last to enter.

Similar as Deng and Papadimitriou (1994) represented undirected networks as a special class of TU-games, we can represent directed networks as generalized TU-games where the worth of ordered coalitions is determined by the two player arcs, i.e. assign to every directed network $\omega$ the generalized characteristic function $v^{\omega}$ given by $v^{\omega}(T)=$ $\sum_{i_{k}, i_{l} \in\left\{i_{1}, i_{2}, \ldots, i_{t}\right\}, k>l} \omega\left(i_{k}, i_{l}\right)$ where $T=\left(i_{1}, i_{2}, \ldots, i_{t}\right)$. Applying $\psi^{N R}$ to this game yields a multiple of the outdegree of $\omega .^{7}$ The solution $\psi^{S B}$ applied to $v^{\omega}$ yields a multiple of the sum of the outdegree and indegree. Applying the class of solutions containing both of van den Brink et al. (2014) gives other centrality measures for directed networks.

\section{Appendix B: Proofs of Section 4}

In this appendix, we give the proofs of Section 4. Proofs of Section 3 follow in a similar way, but also follow from modifying the results in Roth (1977a).

\section{ProOF of Proposition 2}

It is straightforward to verify that centrality/power measures as given by (8) satisfy the four properties. To show uniqueness, suppose that centrality measure $f$ satisfies the four properties, and consider $\omega \in \mathcal{W D}^{N}$.

First, consider the empty network $\omega^{0}$ and any network $\omega \in \mathcal{W D}^{N}$. Additivity implies that $f_{i}\left(\omega+\omega^{0}\right)=f_{i}(\omega)+f_{i}\left(\omega^{0}\right)$. Since $\omega+\omega^{0}=\omega$, this implies that $f_{i}(\omega)=f_{i}(\omega)+f_{i}\left(\omega^{0}\right)$, and thus $f_{i}\left(\omega^{0}\right)=0$ for all $i \in N$. By the isolated node property, $f_{i}(\omega)=0$ for all $\omega \in \mathcal{W D}_{i}^{N}$ where $i$ is isolated.

Next, take a pair $i, j \in N, i \neq j$, and define $\mathcal{W D}_{i j}^{N}=\left\{\omega \in \mathcal{W D}^{N} \mid \omega(i, j) \neq 0\right.$ and $\omega(h, g)=0$ for all $(h, g) \neq(i, j)\}$, being the class of networks where only arc $(i, j)$ has a nonzero weight.

By scale invariance, there exist $\alpha, \beta \in \mathbb{R}$ such that $f_{i}(\omega)=\alpha \cdot \omega(i, j)$ and $f_{j}(\omega)=\beta \cdot \omega(i, j)$ for any $\omega \in \mathcal{W} \mathcal{D}_{i j}^{N}$.

Now take any $(h, g) \in N \times N, h \neq g,(h, g) \neq(i, j)$, and $\omega^{\prime} \in \mathcal{W D}_{h g}^{N}$. By anonymity and the class $\mathcal{W D}_{i j}^{N}$ discussed above, we have $f_{h}\left(\omega^{\prime}\right)=\alpha \cdot \omega^{\prime}(h, g)$ and $f_{g}\left(\omega^{\prime}\right)=\beta \cdot \omega^{\prime}(h, g)$.

Finally, consider any $\omega \in \mathcal{W} \mathcal{G}^{N}$. For every $i, j \in N, i \neq j$, define $\omega^{i j}(i, j)=\omega(i, j)$ and $\omega^{i j}(h, g)=0$ for all $(h, g) \neq(i, j)$. Then additivity implies that for all $i \in N, f_{i}(\omega)=$ $\sum_{\substack{h, g \in N \\ h \neq g}} f_{i}\left(\omega^{h g}\right)=\sum_{j \in N \backslash\{i\}}\left(f_{i}\left(\omega^{i j}\right)+f_{i}\left(\omega^{j i}\right)\right)=\sum_{j \in N \backslash\{i\}}(\alpha \cdot \omega(i, j)+\beta \cdot \omega(j, i))=$ $\alpha \sum_{j \in N \backslash\{i\}}^{h \neq g} \omega(i, j)+\beta \sum_{j \in N \backslash\{i\}} \omega(j, i)=\alpha \cdot$ out $_{i}(\omega)+\beta \cdot i n_{i}(\omega)$.

\footnotetext{
$\overline{7}$ Notice that the game $v^{\omega}$ could also be defined by taking the sum over all ordered pairs $i_{k}, i_{l} \in\left\{i_{1}, i_{2}, \ldots, i_{t}\right\}$ with $k<l$, but then the solution of Nowak and Radzik (1994) will give the indegree of $\omega$.
} 


\section{Proof of Lemma 2}

(i) This follows immediately from Axiom 8.

(ii) If expected utility function $\phi$ represents a preference relation $\succeq$ satisfying Axiom 9 , then for every $\omega, \omega^{\prime} \in \mathcal{W D}_{i}^{N}$, we have $(i, \omega) \sim\left(i, \omega^{\prime}\right)$, and thus $f_{i}(\omega)=\phi(i, \omega)=$ $\phi\left(i, \omega^{\prime}\right)=f_{i}\left(\omega^{\prime}\right)$.

(iii) Consider $\omega \in \mathcal{W} \mathcal{D}^{N}$ and $c>1$. Suppose that $\succeq$ satisfies neutrality to ordinary risk. Taking $p=\frac{1}{c}, \omega^{\prime}=\omega^{0}$ and considering the network $c \omega$, neutrality to ordinary risk implies that

$$
\left(i,\left(\frac{1}{c} c \omega+\left(1-\frac{1}{c}\right) \omega^{0}\right)\right) \sim\left[\frac{1}{c}(i, c \omega) ;\left(1-\frac{1}{c}\right)\left(i, \omega^{0}\right)\right]
$$

which is equivalent to

$$
(i, \omega) \sim\left[\frac{1}{c}(i, c \omega) ;\left(1-\frac{1}{c}\right)\left(i, \omega^{0}\right)\right]
$$

(iv) To show scale invariance, note that from Herstein and Milnor (1953) (see preliminaries) it follows that there exist $r_{0}, r_{1} \in M$ with $r_{1} \succ r_{0}$ such that an expected utility function $\phi$ over the positions in a weighted network $\omega$ can be written as

$$
\phi(i, \omega)=\frac{p_{a b}(i, \omega)-p_{a b}\left(r_{0}\right)}{p_{a b}\left(r_{1}\right)-p_{a b}\left(r_{0}\right)}
$$

for some $a, b \in M$ with $a \succeq(i, \omega) \succeq b$ and $a \succeq r_{1} \succ r_{0} \succeq b$ with probabilities $p_{a b}(i, \omega)$ defined such that $(i, \omega) \sim\left[p_{a b}(i, \omega) a ;\left(1-p_{a b}(i, \omega)\right) b\right]$. By Axiom 9, we can take $b=r_{0}$ and thus $p_{a b}\left(r_{0}\right)=0$ for all $a \in M$. We distinguish the following two cases.

Case 1: Suppose that $(i, c \omega) \succeq r_{1}{ }^{8}$

Take $a=(i, c \omega)$ and $b=r_{0}=\left(i, \omega^{0}\right)$. Then by $(9), \phi(i, c \omega)=\frac{p_{a b}(i, c \omega)}{p_{a b}\left(r_{1}\right)}=\frac{p_{a b}(a)}{p_{a b}\left(r_{1}\right)}=\frac{1}{p_{a b}\left(r_{1}\right)}$. By part (iii) of the lemma, we have $(i, \omega) \sim\left[\frac{1}{c}(i, c \omega) ;\left(1-\frac{1}{c}\right)\left(i, \omega^{0}\right)\right]$, so $p_{a b}(i, \omega)=\frac{1}{c}$. But then $f_{i}(\omega)=\phi(i, \omega)=\frac{p_{a b}(i, \omega)}{p_{a b}\left(r_{1}\right)}=\frac{1}{c} \cdot \frac{1}{p_{a b}\left(r_{1}\right)}=\frac{1}{c} \phi(i, c \omega)=\frac{1}{c} f_{i}(c \omega)$. So, scale invariance is satisfied in this case.

Case 2: Suppose that $r_{1} \succeq(i, c \omega)$.

Take $a=r_{1}$ and $b=r_{0}=\left(i, \omega^{0}\right)$. Then $p_{a b}\left(r_{1}\right)=1$, and so $\phi(i, c \omega)=p_{a b}(i, c \omega)$. By part (iii) of the lemma, we have $(i, \omega) \sim\left[\frac{1}{c}(i, c \omega) ;\left(1-\frac{1}{c}\right)\left(i, \omega^{0}\right)\right] \sim\left[\frac{1}{c}\left[p_{a b}(i, c \omega) a ;(1-\right.\right.$ $\left.\left.\left.p_{a b}(i, c \omega)\right) b\right] ;\left(1-\frac{1}{c}\right)\left(i, \omega^{0}\right)\right]=\left[\frac{1}{c} p_{a b}(i, c \omega) a ; 1-\frac{1}{c} p_{a b}(i, c \omega) b\right]$, so $p_{a b}(i, \omega)=\frac{1}{c} p_{a b}(i, c \omega)$. Then, by (9), we have $\phi(i, \omega)=p_{a b}(i, \omega)=\frac{1}{c} p_{a b}(i, c \omega)$, and thus $f_{i}(\omega)=\phi(i, \omega)=$ $\frac{1}{c} p_{a b}(i, c \omega)=\frac{1}{c} \phi(i, c \omega)=\frac{1}{c} f_{i}(c \omega)$. So, scale invariance is also satisfied in this case.

To prove additivity, consider any $\omega, \omega^{\prime} \in \mathcal{W D}^{N}$. Note that for every $i \in N$, neutrality to ordinary risk implies that $\left(i, \frac{1}{2} \omega+\frac{1}{2} \omega^{\prime}\right) \sim\left[\frac{1}{2}(i, \omega) ; \frac{1}{2}\left(i, \omega^{\prime}\right)\right]$ and thus $\phi\left(i, \frac{1}{2} \omega+\right.$

\footnotetext{
${ }^{8}$ Although Roth (1977a) takes specifically that $r_{1}$ is the unanimity game with player $i$ as only nonnull player, we do not specify $r_{1}$. It is sufficient that there exists a network position that is strictly preferred to being in the empty network, as is guaranteed by Axiom 9 with the center of the outward oriented star network $\left(i, \omega^{i}\right)$.
} 
$\left.\frac{1}{2} \omega^{\prime}\right)=\phi\left(\left[\frac{1}{2}(i, \omega) ; \frac{1}{2}\left(i, \omega^{\prime}\right)\right]\right)=\frac{1}{2} \phi(i, \omega)+\frac{1}{2} \phi\left(i, \omega^{\prime}\right)$, where the first equality follows from neutrality to ordinary risk and the second from (5). But then $f_{i}\left(\omega+\omega^{\prime}\right)=$ $f_{i}\left(2\left(\frac{1}{2} \omega+\frac{1}{2} \omega^{\prime}\right)\right)=2 f_{i}\left(\frac{1}{2} \omega+\frac{1}{2} \omega^{\prime}\right)=2 \phi\left(i, \frac{1}{2} \omega+\frac{1}{2} \omega^{\prime}\right)=2\left(\frac{1}{2} \phi(i, \omega)+\frac{1}{2} \phi\left(i, \omega^{\prime}\right)\right)=\phi(i, \omega)+$ $\phi\left(i, \omega^{\prime}\right)=f_{i}(\omega)+f_{i}\left(\omega^{\prime}\right)$, where the second equality follows from scale invariance of $f$. So, additivity is satisfied.

\section{Proof of TheOREM 2}

To prove the 'only if' part, note that it follows from Lemma 2 and Proposition 2 that, if expected utility function $\phi$ represents a regular preference relation that is neutral to ordinary risk, then $\phi(i, \omega)=\alpha \cdot$ out $_{i}(\omega)+\beta \cdot i n_{i}(\omega)$ for some $\alpha, \beta \in \mathbb{R}$. Then $\phi_{i}\left(\omega^{0}\right)=0$ for all $i \in N$. By Axiom 9(iii), it must hold that $\phi\left(i, \omega^{i}\right)=\alpha(|N|-1)>0=\phi\left(i, \omega^{0}\right)$, and thus $\alpha>0$. By Axiom 9(ii), it must hold that $\phi\left(j, \omega^{i}\right)=\beta \geq 0=\phi\left(j, \omega^{0}\right)$ for all $j \in N \backslash\{i\}$, and thus $\beta \geq 0$.

To prove the 'if' part, let $\succeq$ be the preference relation based on $\phi(i, \omega)=\alpha \cdot$ out $_{i}(\omega)+$ $\beta \cdot i n_{i}(\omega)$ with $\alpha>0, \beta \geq 0$, i.e. $(i, \omega) \succeq\left(j, \omega^{\prime}\right)$ if and only if $\alpha \cdot \operatorname{out}_{i}(\omega)+\beta \cdot i n_{i}(\omega) \geq$ $\alpha \cdot$ out $_{i}\left(\omega^{\prime}\right)+\beta \cdot i n_{i}\left(\omega^{\prime}\right)$. It is straightforward to check that $\succeq$ satisfies Axiom 8. Axiom 9 follows since (i) $\alpha \cdot$ out $_{i}\left(\omega^{\prime}\right)+\beta \cdot$ in $_{i}\left(\omega^{\prime}\right)=0$ for all $\omega^{\prime} \in \mathcal{W} \mathcal{D}_{i}^{N}$, (ii) $\alpha \cdot$ out $_{i}(\omega)+\beta \cdot i n_{i}(\omega) \geq 0$ for all $\omega \in \mathcal{W} \mathcal{D}^{N}$, and (iii) $\alpha \cdot$ out $_{i}\left(\omega^{i}\right)+\beta \cdot i n_{i}\left(\omega^{i}\right)>0$. Finally, to prove neutrality to ordinary risk, consider $\omega, \omega^{\prime} \in \mathcal{W D}^{N}$ and $i \in N$. Then, for $p \in[0,1]$ we have $\phi(i, p \omega+(1-$ $\left.p) \omega^{\prime}\right)=\alpha \cdot$ out $_{i}\left(p \omega+(1-p) \omega^{\prime}\right)+\beta \cdot$ in $_{i}\left(p \omega+(1-p) \omega^{\prime}\right)=\alpha \cdot$ out $_{i}(p \omega)+\alpha \cdot$ out $_{i}\left((1-p) \omega^{\prime}\right)+\beta$. $i n_{i}(p \omega)+\beta \cdot i n_{i}\left((1-p) \omega^{\prime}\right)=p \alpha \cdot$ out $_{i}(\omega)+(1-p) \alpha \cdot$ out $_{i}\left(\omega^{\prime}\right)+p \beta \cdot$ in $_{i}(\omega)+(1-p) \beta \cdot$ in $_{i}\left(\omega^{\prime}\right)=$ $p\left(\alpha \cdot\right.$ out $_{i}(\omega)+\beta \cdot$ in $\left._{i}(\omega)\right)+(1-p)\left(\alpha \cdot\right.$ out $\left._{i}\left(\omega^{\prime}\right)+\beta \cdot i n_{i}\left(\omega^{\prime}\right)\right)=p \phi(i, \omega)+(1-p) \phi\left(i, \omega^{\prime}\right)=$ $\phi\left(\left[p(i, \omega) ;(1-p)\left(i, \omega^{\prime}\right)\right]\right)$, where the last equality follows from $(5)$. 


\section{Bibliography}

J. Banzhaf. Weighted voting doesn't work: a mathematical analysis. Rutgers Law Review, 19:317-343, 1965.

B. Bavelas. A mathematical model for group structure. Human Organizations, 7:16-30, 1948.

B. Bavelas. Communication patterns in task-oriented groups. Journal of the Acoustical Society of America, 22:271-282, 1950.

M. A. Beauchamp. An improved index of centrality. Behavioral Science, 10:161-163, 1965.

F. Bloch, M. O. Jackson, and P. Tebaldi. Centrality measures in networks. Working Paper, 2016.

P. B. Bonacich. Factoring and weighting approaches to status scores and clique identification. Journal of Mathematical Sociology, 2:113-120, 1972.

P. B. Bonacich. Power and centrality: a family of measures. American Journal of Sociology, 92:1170-1182, 1987.

S. P. Borgatti. Centrality and network flow. Social Networks, 27(1):55-71, 2005.

D. Bouyssou. Ranking methods based on valued preference relations: A characterization of the net flow method. European Journal of Operational Research, 60:61-67, 1992.

D. Bouyssou and P. Perny. Ranking methods for valued preference relations: A characterization of a method based on leaving and entering flows. European Journal of Operational Research, 61:186-194, 1992.

D. Brown and D. Housman. Cooperative games on weighted graphs. Internal Report, Worcester Polytechnic Institute, Worcester, MA, 1988.

J. S. Coleman. Control of collectivities and the power of a collectivity to act. In B. Lieberman, editor, Social Choice, pages 269-300. New York: Gordon and Breach, 1971.

A. H. Copeland. A Reasonable Social Welfare Function. Mimeographed Notes, University of Michigan Seminar on Applications of Mathematics to the Social Sciences, 1951.

G. Demange. A ranking method based on handicaps. Theoretical Economics, 9(3):915942, 2014.

G. Demange. Mutual rankings. Mathematical Social Sciences, 2016. Forthcoming.

X. Deng and C. H. Papadimitriou. On the complexity of cooperative solution concepts. Mathematics of Operations Research, 19(2):257-266, 1994.

V. Dequiedt and Y. Zenou. Local and consistent centrality measures in networks. Working paper, submitted, 2014.

P. Dubey. On the uniqueness of the Shapley value. International Journal of Game Theory, 4:131-139, 1975.

P. Dubey and L. S. Shapley. Mathematical properties of the Banzhaf power index. Mathematics of Operations Research, 4:99-131, 1979.

L. Freeman. A set of measures of centrality based on betweenness. Sociometry, 40:35-41, 1977.

L. C. Freeman. Centrality in social networks: Conceptual clarification. Social Networks, 1:215-239, 1979.

M. Garg. Foundations of centrality in networks. Mimeo, 2009. 
D. Gómez, E. González-Arangüena, C. Manuel, G. Owen, M. del Pozo, and J. Tejada. Centrality and power in social networks: a game theoretic approach. Mathematical Social Sciences, 46:27-54, 2003.

S. Goyal. Connections: An Introduction to the Economics of Networks. Princeton University Press, 2007.

J. C. Harsanyi. A bargaining model for the cooperative n-person game. In A. W. Tucker and R. D. Luce, editors, Contributions to the Theory of Games 4, pages 325-335. Princeton, N.J.: Princeton University Press, 1959.

D. Henriet. The Copeland choice function - an axiomatic characterization. Social Choice and Welfare, 2:49-63, 1985.

I. N. Herstein and J. Milnor. An axiomatic approach to measurable utility. Econometrica, 21:291-297, 1953.

M. O. Jackson. Social and Economic Networks. Princeton University Press, 2008.

L. Katz. A new status index derived from sociometric analysis. Psychometrika, 18:39-43, 1953.

M. Kitti. Axioms for centrality scoring with principal eigenvectors. Social Choice and Welfare, 46(3):639-653, 2016.

F. Maniquet. A characterization of the Shapley value in queueing models. Journal of Economic Theory, 109(1):90-103, 2003.

W. Neuefeind and W. Trockel. Continuous linear representability of binary relations. Economic Theory, 6:351-356, 1995.

M. E. J. Newman. Networks: An Introduction. Oxford University Press, 2010.

J. Nieminen. On centrality in a graph. Scandinavian Journal of Psychology, 15:322-336, 1974.

A. S. Nowak and T. Radzik. The Shapley value for n-person games in generalized characteristic function form. Games and Economic Behavior, 6:150-161, 1994.

G. Owen. Multilinear extensions and the Banzhaf value. Naval Research Logistic Quarterly, 22:741-750, 1975.

I. Palacios-Huerta and O. Volij. The measurement of intellectual influence. Econometrica, 72:963-977, 2004.

M. Pintér. On the Axiomatizations of the Shapley Value. Corvinus University of Budapest, 2014.

A. E. Roth. The Shapley value as a von Neumann-Morgenstern utility. Econometrica, 45(3):657-664, 1977a.

A. E. Roth. Bargaining ability, the utility of playing a game, and models of coalition formation. Journal of Mathematical Psychology, 16:153-160, 1977b.

A. E. Roth. Utility functions for simple games. Journal of Economic Theory, 16:481-489, 1977c.

A. E. Roth. A note on values and multilinear extensions. Naval Research Logistics Quarterly, 24:517-520, 1977d.

A. E. Roth. The expected utility of playing a game. In A. E. Roth, editor, The Shapley Value: Essays in Honor of Lloyd S. Shapley, pages 51-70. Cambridge University Press, 1988.

A. Rubinstein. Ranking the participants in a tournament. SIAM Journal of Applied Mathematics, 38(1):108-111, 1980.

G. Sabidussi. The centrality index of a graph. Psychometrika, 31:581-603, 1966. 
E. Sánchez and G. Bergantinos. On values for generalized characteristic functions. O.R. Spektrum, 19:229-234, 1997.

D. Schmeidler. The nucleolus of a characteristic function game. SIAM Journal of Applied Mathematics, 17:1163-1170, 1969.

L. S. Shapley. A value for n-person games. In H. W. Kuhn and A. W. Tucker, editors, Annals of Mathematics Studies, 28, pages 307-317. Princeton University Press, 1953.

L. S. Shapley and M. Shubik. A method for evaluating the distribution of power in a committee system. American Political Science Review, 48:787-792, 1954.

M. E. Shaw. Group structure and the behaviour of individuals in small groups. Journal of Psychology, 38:139-149, 1954.

G. Slutzki and O. Volij. Scoring of web pages and tournaments - axiomatizations. Social Choice and Welfare, 26:75-92, 2006.

S. Tijs. Bounds for the core of a game and the $\tau$-value. In O. Moeschlin and D. Pallaschke, editors, Game Theory and Mathematical Economics, pages 123-132. North-Holland Publ. Comp., Amsterdam, 1981.

W. Trockel. Classification of budget-invariant monotonic preferences. Economics Letters, 30:7-10, 1989.

W. Trockel. An alternative proof for the linear utility representation theorem. Economic Theory, 2:298-302, 1992.

R. van den Brink and R. P. Gilles. Measuring domination in directed networks. Social Networks, 22:141-157, 2000.

R. van den Brink and R. P. Gilles. Ranking by outdegree for directed graphs. Discrete Mathematics, 271:261-270, 2003.

R. van den Brink and R. P. Gilles. The outflow ranking method for weighted directed graphs. European Journal of Operational Research, 193:484-491, 2009.

R. van den Brink, E. González-Arangüena, C. Manuel, and M. del Pozo. Order monotonic solutions for generalized characteristic functions. European Journal of Operational Research, 238:786-796, 2014.

A. van den Nouweland, P. Borm, W. van Golstein Brouwers, R. Groot Bruinderink, and S. Tijs. A game theoretic approach to problems in telecommunication. Management Science, 42(2):294-303, 1996.

J. von Neumann and O. Morgenstern. Theory of Games and Economic Behavior. Princeton: Princeton University Press, 1944. 